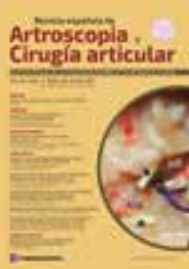

\title{
Original
}

\section{Lesiones del Labrum acetabular en el choque femoroacetabular}

\author{
J. Más, J. Sanz-Reig, C.M. a Verdú, D. Bustamante, M. Morales, E. Martínez \\ Traumatología Vistahermosa. Clínica Vistahermosa. Alicante, España
}

Correspondencia:

Dr. Jesús Más Martínez

Recibido el 22 de noviembre de 2016

Correo electrónico: jmas@traumavist.com

Aceptado el 15 de febrero de 2017

Disponible en Internet: junio 2017

\section{RESUMEN}

Objetivo: determinar la prevalencia y los factores preoperatorios asociados a la lesión del labrum acetabular en una serie única de pacientes intervenidos mediante cirugía artroscópica de cadera.

Material y metodología: se realizó un estudio prospectivo de los hallazgos artroscópicos de 163 caderas, en 120 pacientes, con diagnóstico de choque femoroacetabular (CFA) intervenidos entre enero de 2012 y septiembre de 2016. Se determinó la prevalencia, la localización, la clasificación y los factores preoperatorios asociados a la lesión del labrum acetabular. Las lesiones del labrum acetabular se clasificaron según la clasificación propuesta por el grupo Multicenter Arthroscopic Hip Outcome Research Network (MAHORN). La edad media de los pacientes fue de 38 años y el grado de degeneración articular fue Tönnis 0 , en 104 caderas, y Tönnis 1, en 59 caderas.

Resultados: artroscópicamente, el tipo de CFA era mixto en 86 caderas (52,7\%), de tipo Cam en 67 (41,1\%) y de tipo Pincer en 10 (6,2\%). El labrum presentaba una morfología normal en 140 caderas (85,9\%), era hipoplásico en 17 (9,8\%) e hiperplásico en 6 (4,3\%). Se detectó algún grado de rotura del labrum en 133 caderas (81,6\%). La localización de las lesiones fue superior y anterior. Conclusión: en nuestro estudio no hemos encontrado factores preoperatorios del paciente que tengan relación con la presencia de lesión del labrum acetabular durante el procedimiento de la artroscopia de cadera.

Nivel de evidencia: nivel IV.

Palabras clave: Labrum acetabular. Artroscopia de cadera. Cadera. Choque femoroacetabular.

\section{ABSTRACT \\ Acetabular labrum injuries in femoroacetabular impingement}

Objective: our purpose was to determine the prevalence rate, location, classification, and factors associated with labrum injuries in patients undergoing hip arthroscopy.

Material and methodology: we performed a prospective study of intraoperative data of 163 hips, in 120 patients, treated with hip arthroscopy for femoroacetabular impingement (FAI) from January 2012 to September 2016. We estimated the prevalence rate, location, classification, and preoperative factors associated with labrum injuries. Multicenter Arthroscopic Hip Outcome Research Network (MAHORN) classification were used for labrum injuries. The mean age of the patients was 38 years old. Tönnis grade was 0 in 104 hips, and 1 in 52 hips.

Results: arthroscopically, FAl was combined in 86 hips (52,7\%), Cam type in 67 (41,1\%), and Pincer type in 10 (6,2\%). The labrum morphology was normal in 140 hips (85,9\%), hipoplastic labrum in 17 (9,8\%), and hyperplastic labrum in 6 hips (4,3\%). Labrum injuries were present in 133 hips $(81,6 \%)$. The location of the lesions was superior-anterior.

Conclusion: in our study, we did not find any preoperative factors related to the presence of acetabular labrum lesions during the hip arthroscopy procedure.

Level of evidence: level IV.

Key words: Acetabular labrum. Hip arthroscopy. Hip. Femoroacetabular impingement.

https://doi.org/10.24129/j.reaca.24158.fs1507026

FS $\odot 2017$ Fundación Española de Artroscopia. Publicado por Imaidea Interactiva en FONDOSCIENCE ${ }^{\circledR}$ (www.fondoscience.com) Este es un artículo Open Access bajo la licencia CC BY-NC-ND (www.creativecommons.org/licenses/by-nc-nd/4.0/). 


\section{Introducción}

El choque femoroacetabular (CFA) es un sindrome clínico que presenta alteraciones de la morfología y la orientación de la cadera que producen un conflicto de espacio entre la cabeza femoral y el acetábulo. Es el movimiento de la articulación de la cadera con la actividad diaria o deportiva el que produce el choque repetitivo de dichas estructuras, que conduce a una lesión del labrum y del cartílago articular ${ }^{(1)}$. Los estudios biomecánicos han demostrado la importancia del labrum en el mantenimiento de la biomecánica articular normal(2). Su efecto de sellado impide que el líquido sinovial se salga de la articulación, manteniendo presiones negativas intraarticulares; proporcionando estabilidad frente a la distracción, rotación y traslación; y reduciendo la fricción sobre el cartílago acetabular $r^{(3,4)}$.

Los pacientes con lesión del labrum refieren dolor en la ingle, que se incrementa con la actividad deportiva, al sentarse o bajar escaleras y que hasta en un $61 \%$ suele ser insidioso ${ }^{(5)}$. Diferentes estudios publicados ${ }^{(6,7)}$ refieren una prevalencia entre el 22 y el 59\%. La importancia de su diagnóstico y tratamiento se debe a la alta asociación que presenta con la lesión cartilaginosa ${ }^{(5)}$. Para determinar patología intraarticular de la cadera, la resonancia nuclear magnética (RNM) es la prueba de elección ${ }^{(8)}$ y se recomienda incrementar la precisión en su diagnóstico mediante el uso de artro- RNM $^{(9)}$ o incluso la artro-RNM en tracción $\mathrm{n}^{(10,11)}$. Nos planteamos la hipótesis de que existen factores preoperatorios en el CFA relacionados con la presencia de lesión del labrum acetabular, lo cual podría ayudar al cirujano ortopédico sobre la indicación de la cirugía artroscópica de cadera, el momento de realizarla y su resultado a medio y largo plazo. El objetivo del presente trabajo fue determinar la prevalencia y los factores preoperatorios asociados a la lesión del labrum acetabular en una serie única de pacientes intervenidos mediante cirugía artroscópica de cadera.

\section{Material y metodología}

Entre enero de 2012 y septiembre de 2016 seleccionamos los pacientes de nuestra base de datos prospectiva de pacientes intervenidos mediante cirugía artroscópica que cumplían los siguientes criterios de inclusión: edad menor de 50 años, diagnóstico de CFA, estadio Tönnis 0-1, altura articular mayor de $2 \mathrm{~mm}$. Se excluyó a los pacientes con enfermedad de Legg-Calve-Perthes, cirugía previa de cadera, necrosis avascular y edad menor de 18 años. Todos los pacientes dieron su consentimiento informado para la inclusión en el estudio y el estudio fue aprobado por el Comité Ético de Investigación Clínica (CEIC) de nuestra institución.

La valoración clínica preoperatoria se realizó mediante la escala modificada de Harris (mHHS) ${ }^{(12)}$, cuestionarios de valoración de la cadera con los apartados para actividades de la vida diaria (HOS-AVD) y para la práctica de deportes (HOS-SSS)(13) traducidos al español y validados $^{(14)}$, así como el cuestionario IHOT12(15).

Radiológicamente, se solicitó proyección anteroposterior de pelvis en bipedestación y proyección axial de Dunn para determinar la presencia de lesión de tipo Cam o Pincer, ángulo alfa, ángulo centro-borde, ángulo de Tönnis ${ }^{(16)}$, altura de la interlínea articular en la zona de carga ${ }^{(17)}$ y grado de degeneración articular según la escala de Tönnis ${ }^{(18)}$. En todos los pacientes se solicitó estudio de RNM convencional en dispositivo 3T. Se incluyeron en el estudio 163 caderas de 120 pacientes. La Tabla 1 muestra los datos generales de la serie.

El paciente era preguntado por el deporte que realizaba y, si practicaba más de uno, se anotaba el predominante. Se consideraron deportes de contacto el fútbol, el baloncesto, las artes marciales y el balonmano. El nivel de actividad física preoperatorio se determinó según la escala de Tegner, con 11 niveles diferentes de menor a mayor grado de actividad (0-10)(19). Ciento cinco casos $(64,4 \%)$ practicaban deportes de contacto. El nivel de actividad física según la clasificación de Tegner era igual o mayor de 6 en 119 casos (73\%) (Tabla 2).

Las cirugías se realizaron con anestesia espinal, según técnica quirúrgica descrita previamente ${ }^{(20)}$. Las lesiones del labrum acetabular se clasificaron según la clasificación propuesta por el grupo Multicenter Arthroscopic Hip Outcome Research Network (MAHORN) (21), que establece 4 categorias: labrum normal, labrum hipoplásico/hiperplásico, rotura del labrum y lesiones intrasustancia del labrum. La rotura del labrum se subclasificaba en rotura compleja degenerativa, separación condrolabral, rotura parcial, rotura completa y lesión de tipo flap, y las lesiones intrasustancia se subcla- 
Tabla 1. Datos generales de la población estudiada

\begin{tabular}{|c|c|c|c|}
\hline \multicolumn{2}{|l|}{ Variable } & Media \pm desviación tipica (rango) & $\%$ \\
\hline \multicolumn{2}{|l|}{ Edad (años) } & $38 \pm 7(18-50)$ & \\
\hline \multicolumn{2}{|l|}{ Peso (kg) } & $72,3 \pm 15,4(48-115)$ & \\
\hline \multicolumn{2}{|l|}{ Talla (m) } & $1,7 \pm 0,09(1,5-1,9)$ & \\
\hline \multicolumn{2}{|c|}{ Îndice de masa corporal (IMC) } & $24,0 \pm 3,6(18,5-34,0)$ & \\
\hline \multicolumn{2}{|l|}{$I M C \geq 30$} & 16 & $9,8 \%$ \\
\hline \multirow{2}{*}{ Sexo } & Hombres & 118 & $72,4 \%$ \\
\hline & Mujeres & 45 & $27,6 \%$ \\
\hline \multirow{2}{*}{ Lado } & Derecho & 89 & $54,6 \%$ \\
\hline & Izquierdo & 74 & $45,4 \%$ \\
\hline \multicolumn{2}{|l|}{ Bilateral } & 43 & $26,4 \%$ \\
\hline \multicolumn{2}{|c|}{ Tiempo evolución (meses) } & $21,3 \pm 18,4(3-99)$ & $25,8 \%$ \\
\hline \multicolumn{2}{|c|}{ Lesión labrum en RNM } & 42 & $2,4 \%$ \\
\hline \multicolumn{2}{|c|}{ Lesión cartilaginosa acetabular en RNM } & 4 & \\
\hline \multicolumn{2}{|c|}{ mHHS preoperatorio } & $75,6 \pm 11,3(14,8-87,9)$ & \\
\hline \multicolumn{2}{|c|}{ HOS-AVD preoperatorio } & $64,2 \pm 19,8(11,8-82,1)$ & \\
\hline \multicolumn{2}{|c|}{ HOS-SSS preoperatorio } & $39,0 \pm 25,7(2,7-83,5)$ & \\
\hline \multicolumn{2}{|c|}{ IHOT12 preoperatorio } & $38,5 \pm 20,7(2,0-81,2)$ & \\
\hline \multicolumn{2}{|c|}{ Ángulo alfa (grados) } & $61,4 \pm 8,9(35-84)$ & \\
\hline \multicolumn{2}{|c|}{ Ángulo alfa $\geq 55^{\circ}$} & 132 & $81 \%$ \\
\hline \multicolumn{2}{|c|}{ Altura articular (mm) } & $4,8 \pm 0,7(2,9-7,0)$ & \\
\hline \multicolumn{2}{|c|}{ Ángulo centro-borde (grados) } & $33,7 \pm 7,2(18-48)$ & \\
\hline \multirow{3}{*}{$\begin{array}{l}\text { Ángulo } \\
\text { centro-borde }\end{array}$} & $<25^{\circ}$ & 12 & $7,3 \%$ \\
\hline & $25-35^{\circ}$ & 101 & $61,9 \%$ \\
\hline & $<35^{\circ}$ & 40 & $30,8 \%$ \\
\hline \multicolumn{2}{|c|}{ Ángulo Tönnis (grados) } & $8,0 \pm 3,3(1-15)$ & \\
\hline \multirow{2}{*}{ Ángulo Tönnis } & $0-10^{\circ}$ & 141 & $86,5 \%$ \\
\hline & $>10$ & 22 & $13,5 \%$ \\
\hline \multirow{2}{*}{ Grado Tönnis } & 0 & 104 & $63,8 \%$ \\
\hline & 1 & 59 & $36,2 \%$ \\
\hline
\end{tabular}

HOS: Hip Outcome Score; IHOT: International Hip Outcome Tool; mHHS: Harris Hip Scores modificado

El análisis estadístico se realizó con el programa SPSS, versión 15.0 (SPSS Inc., Chicago, USA). Para un mejor manejo estadístico de los datos, el tiempo de evolución de los sintomas hasta la cirugía se agrupó en menor o igual y mayor de 20 meses; el ángulo alfa en menor o igual y mayor de $55^{\circ}$; y el nivel de actividad física en menor 0 igual y mayor de nivel 6 . Se consideraron significativos los valores de $p$ iguales o menores a 0,05. Para determinar la distribución normal se usó la prueba de Kolmogorov-Smirnov. Para comparación de variables continuas se utilizaron las pruebas de t-Student o U de Mann-Whitney, y para variables categóricas mediante la prueba de chi-cuadrado en variables con distribución normal o la de Mantel-Haenszel para datos no paramétricos. Se calculó el riesgo relativo con su intervalo de confianza del 95\% en las variables categóricas preoperatorias significativas asociadas a la presencia de lesión del labrum acetabular.

\section{Resultados}

Artroscópicamente, el tipo de CFA era mixto

sificaban en mucoide o amarillo, blando, hemático, osificado y calcificado. Para la localización de la lesión se utilizó el método geográfico descrito por Illizaliturri et al.(22). en 86 caderas (52,7\%), de tipo Cam en 67 (41,1\%) y de tipo Pincer en 10 (6,2\%). El labrum presentaba una morfología normal (Figura 1) en 140 caderas (85,9\%), hipoplásico (Figura 2) en 17 (9,8\%) e hi- 


\section{Tabla 2. Nivel de actividad física según la escala Tegner}

\begin{tabular}{l|l|c|c}
\hline Nivel & Actividad & $\mathbf{N}$ & $\%$ \\
\hline Nivel 1 & Trabajo sedentario & 10 & 6,1 \\
\hline Nivel 3 & Trabajo liviano, caminar llano & 5 & 3,1 \\
\hline Nivel 4 & $\begin{array}{l}\text { Ocio: ciclismo, carrera 2 v/sem. llano } \\
\text { Trabajo: camionero, doméstico }\end{array}$ & 8 & 4,9 \\
\hline Nivel 5 & $\begin{array}{l}\text { Ocio: carrera 2 v/sem. irregular } \\
\text { Competición: ciclismo }\end{array}$ & 21 & 12,9 \\
\hline Nivel 6 & Ocio: tenis, balonmano, baloncesto, carrera 5 v/sem & 49 & 30,1 \\
\hline Nivel 7 & $\begin{array}{l}\text { Competición: tenis, motocross, atletismo, balonmano, baloncesto } \\
\text { Ocio: squash, fútbol, cross }\end{array}$ & 63 & 38,7 \\
\hline Nivel 8 & Competición: squash, atletismo, bádminton & 1 & 0,6 \\
\hline Nivel 9 & Competición: fútbol, gimnasia, hockey hielo & 3 & 1,8 \\
\hline Nivel 10 & Competición nacional o internacional: fútbol & 3 & 1,8 \\
\hline
\end{tabular}

Las roturas del labrum se localizaron mayoritariamente en la zona 2 y 3 en un $60,1 \%$ (80 caderas), seguido por la zona 2 en un $18,7 \%$ (25 caderas) y la zona 3 en un $11,2 \%$ (15 caderas). En las 133 caderas con rotura del labrum, el 75,1\% (100 caderas) asociaba lesión cartilaginosa. Por el contrario, en las 30 caderas sin rotura franca del labrum, el $56,6 \%$ (17 caderas) asociaba lesión cartilaginosa $(p=0,03)$. El riesgo relativo de asociar una lesión cartilaginosa en presencia de una lesión

perplásico (Figura 3) en 6 (4,3\%). Se detectó algún grado de rotura del labrum en 133 caderas (81,6\%); de ellas, en 98 caderas $(60,1 \%)$ eran separaciones condrolabrales (Figura 4), en $26(15,9 \%)$ roturas complejas-degenerativas (Figura 5), en $5(3,0 \%)$ roturas parciales y en $4(2,4 \%)$ roturas completas. El labrum presentaba cambios intrasustancia en 30 caderas $(18,4 \%)$, distinguiendo en $19(63,3 \%)$ calcificado, 9 (30\%) hemático (Figura 6) y 2 (6,6\%) mucoide-amarillo.

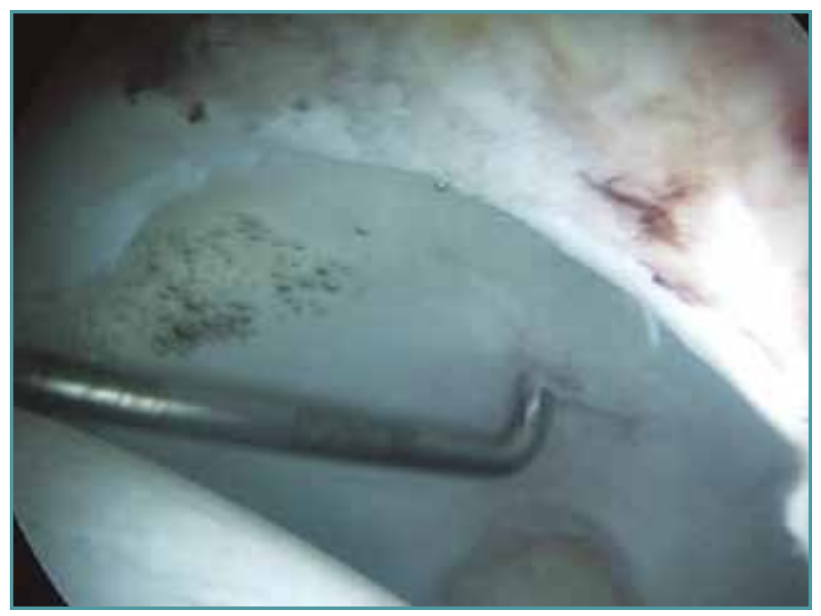

Figura 1. Imagen intraoperatoria de la cadera izquierda desde el portal anterolateral de labrum de morfología normal. Instrumental introducido desde portal medio-anterior. del labrum fue de 2,31 (IC 95\%: 1,01-5,27).

La morfología del labrum no tenía relación significativa con el tiempo de evolución, el tipo de CFA y las mediciones radiológicas. Si bien el número de caderas con labrum hiperplásico era reducido, la medición radiológica mostraba un ángulo alfa mayor, un ángulo centro-borde menor

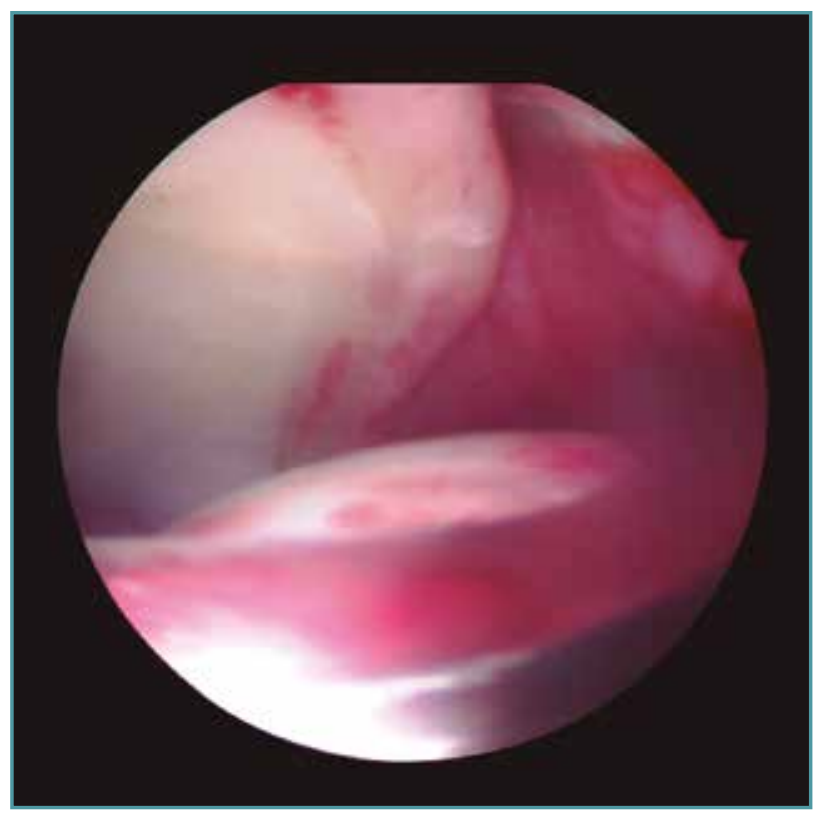

Figura 2. Imagen intraoperatoria de la cadera derecha desde el portal anterolateral de labrum hipoplásico. 


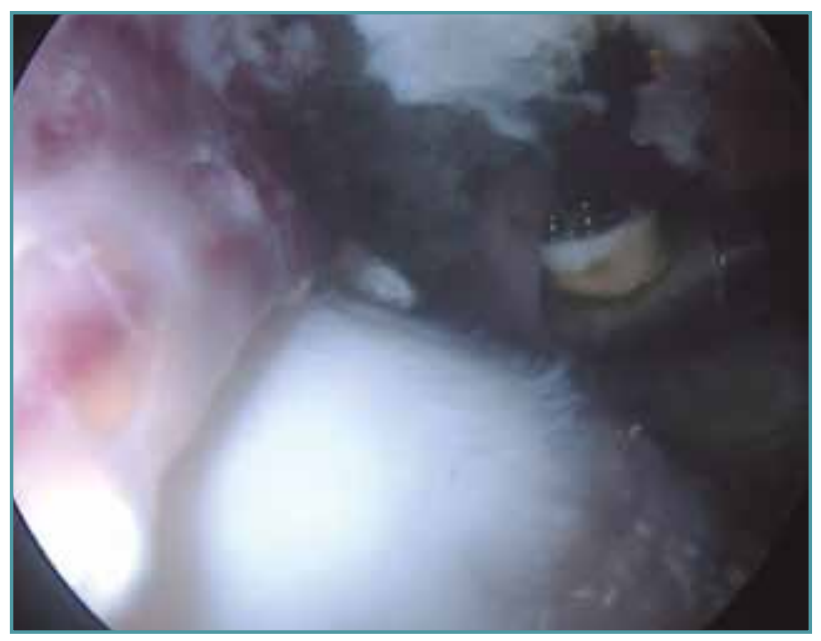

Figura 3. Imagen intraoperatoria de la cadera derecha desde el portal anterolateral de labrum hiperplásico. Instrumental introducido desde portal medio-anterior.

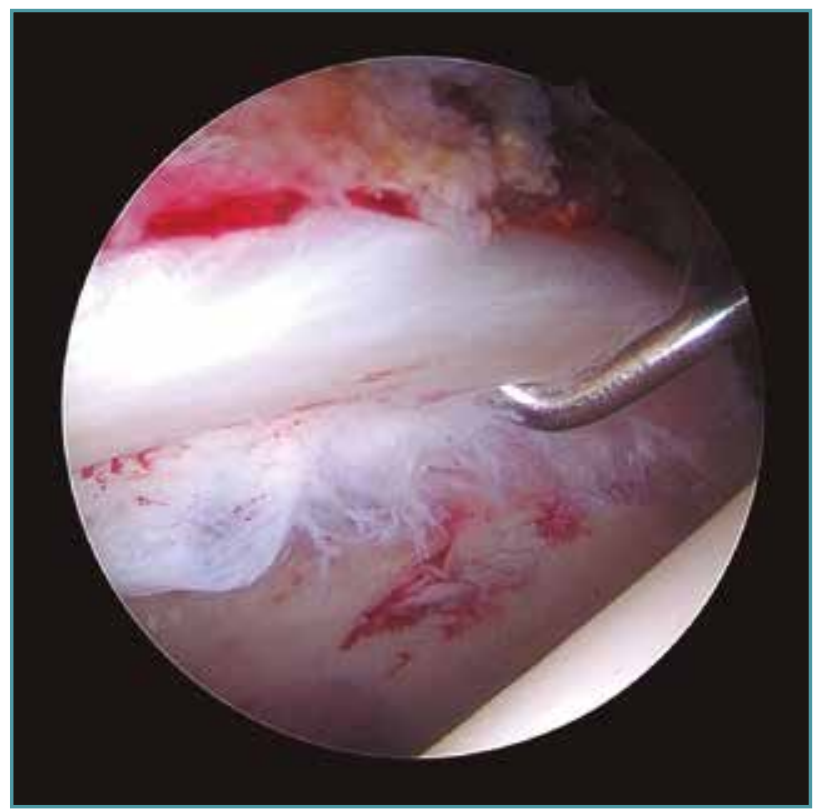

Figura 4. Imagen intraoperatoria de la cadera derecha desde el portal anterolateral con morfología del labrum normal y rotura condrolabral. Instrumental introducido a través del portal medio-anterior.

y un ángulo de Tönnis mayor que las caderas con labrum hipoplásico o labrum normal (Tabla 3).

Los pacientes con rotura del labrum referian un tiempo medio de inicio de los sintomas hasta la cirugía mayor que los pacientes sin rotura, siendo las diferencias significativas $(p=0,05)$. Sin

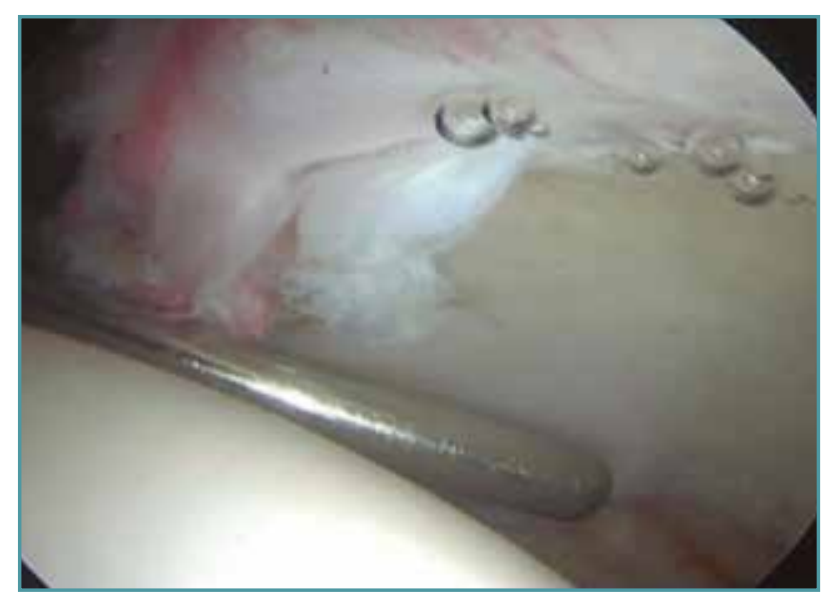

Figura 5. Imagen intraoperatoria de la cadera izquierda desde el portal anterolateral con rotura compleja-degenerativa del labrum acetabular. Instrumental introducido a través del portal medio-anterior.

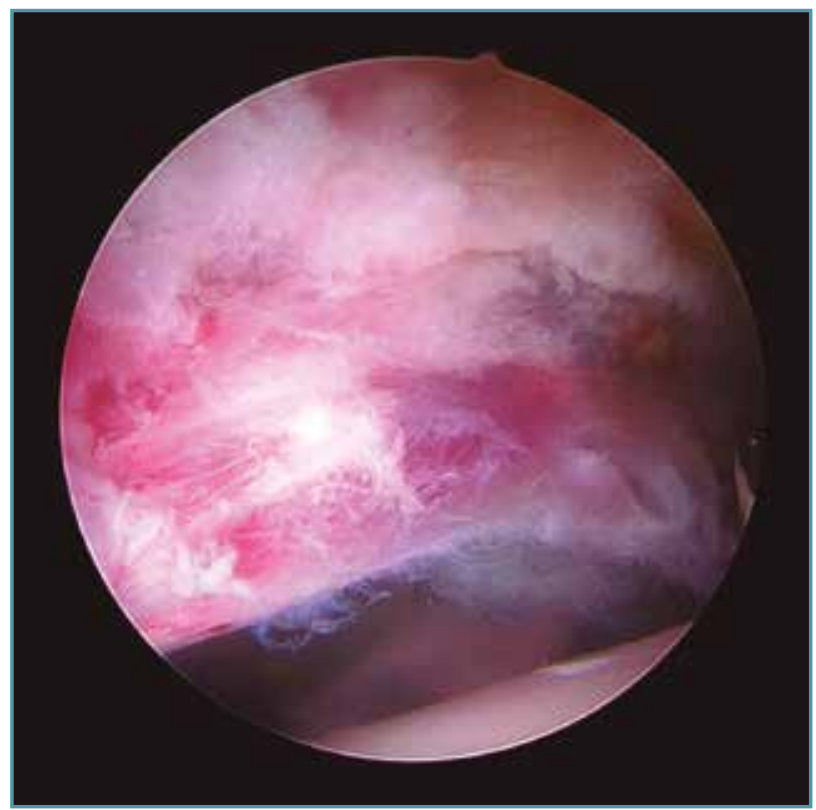

Figura 6. Imagen intraoperatoria de la cadera derecha desde el portal anterolateral con labrum de contenido hemorrágico.

embargo, no había diferencias significativas con respecto a un tiempo de evolución mayor de 20 meses, una actividad física igual o mayor al nivel 6, la práctica de deporte de contacto, los cuestionarios de valoración clínica preoperatoria, el tipo de CFA y las mediciones radiológicas (Tabla 4). 
Tabla 3. Variables preoperatorias y morfología del labrum

\begin{tabular}{|c|c|c|c|c|}
\hline & Normal & Hipoplásico & Hiperplásico & $\mathbf{p}$ \\
\hline & \multicolumn{3}{|c|}{ Media \pm desviación estándar } & \\
\hline & 140 & 17 & 6 & \\
\hline Tiempo evolución (meses) & $22 \pm 19$ & $18 \pm 15$ & $19 \pm 15$ & 0,75 \\
\hline Ángulo alfa $\left(^{\circ}\right)$ & $61,9 \pm 8,3$ & $56,8 \pm 12,1$ & $64,7 \pm 10,2$ & 0,06 \\
\hline Altura articular (mm) & $4,9 \pm 0,7$ & $4,5 \pm 0,6$ & $4,6 \pm 0,5$ & 0,06 \\
\hline Ángulo centro-borde $\left(^{\circ}\right)$ & $33,8 \pm 7,3$ & $34,0 \pm 6,9$ & $33,0 \pm 9,0$ & 0,97 \\
\hline Ángulo Tönnis (º) & $8,0 \pm 3,4$ & $7,2 \pm 2,7$ & $11,0 \pm 1,4$ & 0,61 \\
\hline CFA mixto & 74 & 10 & 2 & \multirow{3}{*}{0,57} \\
\hline CFA de tipo Cam & 63 & & 4 & \\
\hline CFA de tipo Pincer & 3 & 7 & & \\
\hline
\end{tabular}

CFA: choque femoroacetabular

Existen diferentes clasificaciones de las lesiones del labrum ${ }^{(24)}$. El grupo MAHORN propuso una clasificación para determinar si el resultado del tratamiento es diferente según el tipo de lesión labral(21). El objetivo del presente manuscrito era realizar una descripción de las lesiones del labrum, sin referir el tipo de tratamiento aplicado ni el resultado del mismo. En nuestra opinión, la clasificación propuesta por el grupo MAHORN permite determinar la morfología del labrum, el tipo de rotura y la pre-

\section{Discusión}

Los hallazgos más importantes de nuestro estudio fueron en una serie única de pacientes con diagnóstico de CFA y estudio radiológico Tönnis 0 y 1; se detectó algún grado de lesión del labrum en un $81,6 \%$ durante la cirugía artroscópica; la presencia de una rotura labral durante la cirugía artroscópica asociaba un riesgo relativo de 2,31 para la presencia de lesión cartilaginosa asociada y no hemos encontrado factores predictores preoperatorios de la lesión del labrum, por lo cual debemos rechazar nuestra hipótesis inicial.

La RNM con contraste y distensión capsular (artro-RNM) constituye la prueba de imagen de elección para la detección de la lesión del labrum, con una sensibilidad entre el 62 y el $86 \%$, en comparación con los hallazgos intraoperato$\operatorname{rios}^{(23,24)}$, aunque se recomienda realizar la prueba diagnóstica con tracción para incrementar la sensibilidad y especificidad ${ }^{(10,11)}$. Sin embargo, presenta el inconveniente de ser una prueba invasiva, consumir mayor tiempo para su ejecución y, en ocasiones, los pacientes refieren un dolor inguinal residual que persiste durante tiempo variable. La RNM convencional tiene una baja sensibilidad para la detección de la lesión del labrum ${ }^{(23,25)}$. En nuestra serie se informó de lesión del labrum en un $25,8 \%$ de los casos. sencia de cambios intrasustancia, y, de este modo, se puede establecer el mecanismo subyacente que ha causado las lesiones.

En nuestra serie de pacientes con un $73 \%$ realizando una actividad física de nivel $\geq 6$ según Tegner, la lesión del labrum se informó en un 25,8\% de los casos según la RNM, que se incrementó a un $81,6 \%$ durante el procedimiento de la cirugía artroscópica, dato superior a otras series descritas previamente $e^{(5,7,26)}$.

La morfología del labrum normal ha predominado en el CFA mixto y en el CFA de tipo Cam, en un 86 y un $94 \%$, respectivamente. El sobrecrecimiento o "giba" presente en la zona de transición entre la cabeza y el cuello femoral provoca, en los movimientos de flexión y rotación interna de la cadera, una elevación e impacto sobre el labrum, que afecta a la unión condrolabral $^{(1,5)}$. El labrum hipoplásico se ha presentado en el $41,1 \%$ de las caderas con CFA de tipo Pincer. El mecanismo de choque de tipo "contrafuerte" entre el cuello femoral y el labrum provoca un aplastamiento continuado del labrum acetabular que causa la degeneración y la hipoplasia ${ }^{(1,4)}$ y el labrum hiperplásico ha predominado en CFA de tipo Cam con valoración radiológica indicativa de déficit de cobertura acetabular, en las que debido a esta falta de contención ósea, las fuerzas de carga actúan directamente sobre el labrum acetabular ${ }^{(27)}$. 
Tabla 4.Variables preoperatorias y rotura del labrum

\begin{tabular}{|c|c|c|c|}
\hline & Si & No & \multirow[t]{3}{*}{$\mathbf{p}$} \\
\hline & \multicolumn{2}{|c|}{ Media \pm desviación estándar } & \\
\hline & 133 & 30 & \\
\hline Tiempo evolución (meses) & $22 \pm 19$ & $18 \pm 18$ & 0,05 \\
\hline Tiempo evolución $\geq 20$ meses & 58 & 9 & 0,12 \\
\hline Tegner $\geq 6$ & 96 & 23 & 0,82 \\
\hline Deporte contacto & 85 & 20 & 0,47 \\
\hline mHHS preoperatorio & $75,8 \pm 11,1$ & $74,6 \pm 12,3$ & 0,97 \\
\hline HOS-AVD preoperatorio & $63,0 \pm 20,1$ & $70,4 \pm 17,0$ & 0,45 \\
\hline HOS-SSS preoperatorio & $40.0 \pm 24,6$ & $45,0 \pm 25,6$ & \\
\hline IHOT12 preoperatorio & $37,8 \pm 21,0$ & $42,6 \pm 19,3$ & \\
\hline Ángulo alfa $\left({ }^{\circ}\right)$ & $61,9 \pm 8,5$ & $59,7 \pm 10,6$ & 0,35 \\
\hline Ángulo alfa $\geq 55^{\circ}$ & 108 & 24 & 0,52 \\
\hline Altura articular (mm) & $4,8 \pm 0,7$ & $4,9 \pm 0,8$ & 0,51 \\
\hline Ángulo centro-borde $\left({ }^{\circ}\right)$ & $33,8 \pm 7,5$ & $33,4 \pm 5,7$ & 0,17 \\
\hline Ángulo centro-borde $<25^{\circ}$ o $>35^{\circ}$ & 44 & 8 & 0,32 \\
\hline Ángulo Tönnis $\left(^{\circ}\right)$ & $7,9 \pm 3,2$ & $8,4 \pm 3,8$ & 0,75 \\
\hline CFA mixto & 68 & 18 & \multirow{3}{*}{0,49} \\
\hline CFA de tipo Cam & 60 & 7 & \\
\hline CFA de tipo Pincer & 8 & 2 & \\
\hline
\end{tabular}

CFA: choque femoroacetabular; HOS: Hip Outcome Score; IHOT: International Hip Outcome Tool; mHHS: Harris Hip Scores modificado

fútbol, hockey, golf, ballet o correr con la lesión labral(5). En nuestra serie la mayoría de los pacientes practicaban una actividad deportiva de alto nivel, lo cual, unido a la elevada prevalencia de la lesión labral, pueda ser la causa de la no relación significativa entre el nivel y el tipo de actividad deportiva, y la lesión del labrum acetabular.

La importancia del diagnóstico y el tratamiento de la lesión del labrum acetabular radica en su elevada asociación con la lesión cartilaginosa. McCarhty et al. ${ }^{(29)}$ publicaron que en un $73 \%$ de los pacientes con lesión labral asociaban lesión cartilaginosa y que, en un $94 \%$ de ellos, la localización de la lesión labral y cartilaginosa coincidían. Y sugerían que el riesgo relativo para la presencia de lesión cartilaginosa se duplicaba en presencia de una lesión labral. Neumann et al. ${ }^{(30)}$

La rotura del labrum acetabular se ha presentado en un 79\% en el CFA mixto, en un 89,5\% en el CFA de tipo Cam y en un $80 \%$ en el CFA de tipo Pincer. Su localización más frecuente es la anterosuperior $^{(4,28)}$, al igual que coincide con los resultados de nuestra serie. La superficie cóncava del acetábulo está más expuesta a las fuerzas de cizallamiento que la cabeza femoral. Y el impacto repetido de la "giba" femoral y el cuello femoral contra el labrum produce una lesión progresiva del mismo.

La actividad deportiva se relaciona con la lesión del labrum acetabular, especialmente en deportes que requieran posiciones de hiperabducción, hiperextensión, hiperflexión y rotación externa. En pocas ocasiones, el paciente relaciona el dolor en la cadera con un traumatismo durante la práctica deportiva. Numerosos estudios relacionan la práctica de refirieron que 9/10 pacientes con lesión del labrum asociaban lesión cartilaginosa. En nuestra serie, un $75,1 \%$ de los pacientes con lesión labral han asociado lesión cartilaginosa y el riesgo relativo para la presencia de lesión cartilaginosa asociada a la lesión labral fue de 2,31.

En nuestro estudio no hemos encontrado factores preoperatorios del paciente que tengan relación con la presencia de lesión del labrum acetabular durante el procedimiento de la cirugía artroscópica.

\section{Bibliografía}

1. Leunig M, Beaulé P, Ganz R. The concept of femoroacetabular impingement: current status and future perspectives. Clin Orthop Relat Res. 2009;467:616-22. 
2. Safran MR. The acetabular labrum: anatomic and functional characteristics and rationale for surgical intervention. J Am Acad Orthop Surg 2010;18:338-45.

3. Nepple JJ, Philippon MJ, Campbell KJ, Dornan GJ, Jansson KS, Laprade RF, et al. The hip fluid seal-Part II: The effect of an acetabular labral tear, repair, resection, and reconstruction on hip stability to distraction. Knee Surg Sports Traumatol Arthrosc. 2014;22:730-6.

4. Beaulé PE, ONeill M, Rakhra K. Acetabular labral tears. J Bone Joint Surg (Am). 2009;91-A:701-10.

5. Groh MM, Herrera J. A comprehensive review of hip labral tears. Curr Rev Musculoskelet Med. 2009;2:105-17.

6. Burnett RS, Della Rocca GJ, Prather H, Curry M, Maloney WJ, Clohisy JC. Clinical presentation of patients with tears of the acetabular labrum. J Bone Joint Surg (Am). 2006;88-A:1448-57.

7. Meerrmans G, Konan S, Haddad F, Witt JD. Prevalence of acetabular cartilage lesions and labral tears in femoroacetabular impingement. Acta Orthop Belg. 2010;76:181-8.

8. Bitthersoll B, Hosalkar HS, Hesper T, Tiderius CJ, Zilkens C, Krauspe R. Advanced imaging in femoroacetabular impingement: current state and future concepts. Front Surg. 2015;24:34.

9. Casado Verdugo OL, Sánchez Sobrino A, Mediavilla Arza I. Pruebas de imagen y evaluación de la patología articular y periarticular de la cadera. Rev Esp Artrosc Cir Articul. 2016;23:19-30.

10. Schmaranzer F, Klauser A, Kogler $M$, Henninger B, Reichkendler M, Schmaranzer E. Diagnostic performance of direct traction MR arthrography of the hip: detection of chondral and labral lesions with arthroscopic comparison. Eur Radiol. 2014;25:1721-30.

11. Llopis E, Cerezal L, Kassarjian A, Higueras V, Fernandez E. Direct MR arthrography of the hip with leg traction: feasibility for assessing articular cartilage. AJR Am J Roentgenol. 2008;190:1124-8.

12. Byrd JW, Jones KS. Prospective analysis of hip arthroscopy with 2-year follow-up. Arthroscopy. 2000;16:578-87.

13. Martin RL, Philippon MJ. Evidence of validity for the Hip Outcome Score in hip arthroscopy. Arthroscopy. 2007;23:822-6.

14. Seijas R, Sallent A, Ruiz-Iban MA, Ares O, Marín-Peña O, Cuellar R, et al. Validation of the Spanish version of the Hip Outcome Score: a multicenter study. Health Qual Life Outcomes. 2014;12:70.

15. Griffin DR, Parsons N, Mohtadi NG, Safran MR. A short version of the International Hip Outcome Tool (iHOT-12) for use in routine clinical practice. Arthroscopy. 2012;28:611-6.

16. Marín O (dir.). Choque femoroacetabular. Madrid: Fundación Mapfre. Ediciones Díaz de Santos; 2010.
17. Philippon MJ, Briggs KK, Yen YM, Kuppersmith DA. Outcomes following hip arthroscopy for femoroacetabular impingement with associated chondrolabral dysfunction. Minimum two-year follow-up. J Bone Joint Surg (Am). 2009;91-A:16-23.

18. Tönnis D. Normal values of the hip joint for the evaluation of $x$-rays in children and adults. Clin Orthop Relat Res. 1976;119:39-47.

19. Tegner $Y$, Lisholm J. Rating systems in the evaluation of knee ligament injuries. Clin Orthop Rel Res. 1985;198:43-9.

20. Más Martínez J, Morales-Santías M, Bustamante Suarez de Puga D, Sanz-Reig J. La cirugía artroscópica de cadera en deportistas varones menores de 40 años con choque femoroacetabular: resultado a corto plazo. Rev Esp Cir Ortop Traumatol. 2014;58:343-50.

21. Safran MR, Hariri S. Hip arthroscopy assessments tools and outcomes. Oper Tech Ortop. 2010;20:264-77.

22. Ilizaliturri VM, Byrd T, Sampson TG, Guanche CA, Philippon MJ, Kelly BT, et al. A geographic zone method to describe intraarticular pathology in hip arthroscopy: cadaveric study and preliminary report. Arthroscopy. 2008;24:534-9.

23. Smith TO, Simpson M, Ejindu V, Hing CB. The diagnostic test accuracy of magnetic resonance imaging, magnetic resonance arthrography and computer tomography in the detection of chondral lesions of the hip. Eur J Orthopaedic Surgery Traumatol. 2013;23:335-44.

24. Freehill MT, Safran MR. The labrum of the hip: diagnosis and rationale for surgical correction. Clin Sports Med. 2011;30:293-315.

25. Naraghi A, White LM. MRI of labral and chondral lesions of the hip. AJR. 2015;205:479-90.

26. Pérez Carro L, Cruz A, Más J, Miranda V, Ortiz A, Alfonso A. Lesiones del labrum de cadera: vascularización y técnicas de reconstrucción. Rev Esp Artrosc Cir Articul. 2016;23:37-46.

27. Hartig-Andreasen C, S $\phi$ balle K, Troelsen A. The role of the acetabular labrum in hip displasia. A literature overview. Acta Orthopaedica. 2013;80:60-4.

28. Imam JH, Khanduja V. Current concepts in the diagnosis and management of femoroacetabular impingement. Int Orthop. 2011;35:1427-35.

29. McCarthy JC, Noble PC, Schuck MR, Wright J, Lee J. The Otto E Aufranc Award: the role of labral lesions to development of early degenerative hip disease. Clin Orthop Rel Res. 2001;393:25-37.

30. Neumann G, Mendicuti A D, Zou K H, Minas T, Coblyn J, Winalski CS, Lang P. Prevalence of labral tears and cartilage loss in patients with mechanical symptoms of the hip: Evaluation using MR arthrography. Osteoarthritis Cartilage. 2007;15:909-7. 